\title{
Diferenciación genética de tres poblaciones colombianas de Triatoma dimidiata (Latreille, 1811) mediante análisis molecular del gen mitocondrial ND4
}

\author{
Nelson Grisales ${ }^{1 \dagger}$, Omar Triana1, Víctor Angulo³, Nicolás Jaramillo, Gabriel Parra-Henao², \\ Francisco Panzera ${ }^{4}$, Andrés Gómez-Palacio ${ }^{1 \dagger}$ \\ 1 Grupo de Biología y Control de Enfermedades Infecciosas, Sede de Investigación Universitaria, Instituto de \\ Biología, Universidad de Antioquia, Medellín, Colombia \\ 2 Instituto Colombiano de Medicina Tropical, Universidad CES, Medellín, Colombia \\ ${ }^{3}$ Centro de Investigaciones en Enfermedades Tropicales, Universidad Industrial de Santander, Bucaramanga, \\ Colombia \\ ${ }^{4}$ Sección Genética Evolutiva, Facultad de Ciencias, Universidad de la República, Montevideo, Uruguay \\ † Estos autores aportaron en igual medida al trabajo. \\ El trabajo se llevó a cabo en el Grupo de Biología y Control de Enfermedades Infecciosas, Sede de \\ Investigación Universitaria, Universidad de Antioquia.
}

Introducción. Triatoma dimidiata es el segundo vector más importante de la enfermedad de Chagas en Colombia, después de Rhodnius prolixus. El conocimiento de la composición genética y la diferenciación de poblaciones es fundamental para el adecuado diseño e implementación de estrategias de control y vigilancia vectorial.

Objetivo. Determinar el nivel de variabilidad y diferenciación genética en tres poblaciones colombianas de $T$. dimidiata provenientes de distintas localidades y hábitats, mediante el análisis molecular de un fragmento del gen mitocondrial ND4.

Materiales y métodos. Se analizó el nivel de polimorfismo y la estructura genética de dos poblaciones silvestres de los departamentos de La Guajira $(n=10)$ y Santander $(n=10)$, y de una población intradomiciliaria $(n=15)$ y peridomiciliaria $(n=5)$ del Cesar. Para tal fin, se analizaron las secuencias de nucleótidos de un fragmento del gen mitocondrial ND4.

Resultados. T. dimidiata en Colombia demostró tener gran diversidad genética, tanto a nivel de nucleótidos ( $\pi: 0,034)$ como de haplotipo (Hd: 0,863), además de una significativa estructuración de población $\left(\phi_{\mathrm{ST}}: 0,761\right)$ con un bajo número de migrantes $(\mathrm{Nm}: 0,157)$. Las distancias genéticas y las diferencias en los niveles de variabilidad genética entre las tres poblaciones fueron coherentes con una posible subdivisión de población.

Conclusión. Este trabajo demostró diferenciación genética entre las poblaciones de $T$. dimidiata de La Guajira, Cesar y Santander. Se sugiere una posible relación entre tal subdivisión y algunas características eco-epidemiológicas que posee $T$. dimidiata en el centro-oriente y en el norte de Colombia. Finalmente, este trabajo describe, por primera vez, la utilidad del ND4 como un marcador molecular para el estudio de poblaciones naturales de $T$. dimidiata.

Palabras clave: enfermedad de Chagas, Triatoma, Triatominae, genética de población, polimorfismo genético, NADH deshidrogenasa.

Genetic differentiation of three Colombian populations of Triatoma dimidiata (Heteroptera: Reduviidae) by ND4 mitochondrial gene molecular analysis

Introduction. Triatoma dimidiata is the second most important vector of Chagas disease in Colombia after Rhodnius prolixus. Population genetic studies are essential for the adequate design and implementation of vector control and surveillance strategies.

Objective. The level of genetic variability and population differentiation was surveyed among three Colombian populations of $T$. dimidiata from different geographic locations and ecotopes, using ND4 mitochondrial gene.

Materials and methods. Genetic comparison was made between two wild populations from La Guajira $(n=10)$ and Santander $(n=10)$ provinces, and one intra $(n=15)$ and one peridomiciliary $(n=5)$ population from the Cesar province. The polymorphism frequencies of the ND4 mitochondrial gene sequence were analyzed to deduce population structure based on the 40 samples.

Results. Colombian T. dimidiata showed a high nucleotide (п: 0.034) and haplotype diversity (Hd: $0.863)$, as well as significant population subdivision $\left(\phi_{\mathrm{ST}}: 0.761\right)$ and a low migration rate $(\mathrm{Nm}: 0.157)$. 
Genetic distances and variability differences among populations indicate distinct population subdivision amongst the three provinces.

Conclusion. ND4 proved useful in elucidating the significant genetic differentiation that has occurred among T. dimidiata populations from La Guajira, Cesar and Santander. The analysis suggested a relationship between population subdivision and some eco-epidemiological attributes of this vector from the central eastern and northwestern regions of Colombia.

Key words: Chagas disease, Triatoma, Triatominae; genetics, population; polymorphism, genetics; $\mathrm{NADH}$ dehydrogenase.

La enfermedad de Chagas es una parasitosis que afecta, aproximadamente, 15 millones de personas en Latinoamérica, con 28 millones en riesgo de contraer la infección (1). El agente etiológico de esta enfermedad, Trypanosoma cruzi, es transmitido al humano, principalmente, por las heces de insectos hematófagos de la subfamilia Triatominae (Hemiptera: Reduviidae). Triatoma dimidiata es una especie de amplia distribución geográfica, que va desde México, e incluye a Centroamérica, Ecuador, Perú y Colombia, y se considera el vector principal de T. cruzi en México y en varios países de Centroamérica (2). En Colombia es el segundo vector más importante después de Rhodnius prolixus, debido a sus frecuentes eventos de intrusión domiciliaria y su incidencia en la transmisión de $T$. cruzi al hombre $(1,2)$.

En Colombia, $T$. dimidiata tiene una amplia distribución y está presente en 15 de los 32 departamentos, y ocupa una amplia diversidad de hábitats, incluso en una misma región se puede hallar de manera simultánea en ecótopos silvestres, peridomésticos y domésticos (3-6).

En la costa norte de Colombia, este vector ocupa, principalmente, ecótopos silvestres, concretamente en palmas de la especie Attalea butyracea, y en esta región presenta bajos niveles de colonización a las viviendas $(5,7)$. Por el contrario, en los departamentos de la región andina, como Santander y Boyacá, T. dimidiata representa un alto riesgo epidemiológico, por encontrarse colonizando ambientes domésticos, peridomésticos y silvestres, simultáneamente $(5,6)$.

A pesar de estos antecedentes, es poco lo que se conoce sobre la estructura genética de las poblaciones de $T$. dimidiata en Colombia. Los

\footnotetext{
Correspondencia:

Andrés Gómez-Palacio, Grupo de Biología y Control de Enfermedades Infecciosas, Laboratorio 620, Sede de Investigación Universitaria, Universidad de Antioquia, Calle 62 \# 52-59, Medellín, Colombia. Fax: (+574) 2196520 amgomezpa@gmail.com

Recibido: 15/07/09; aceptado:06/12/09
}

estudios moleculares con marcadores nucleares, que usan RAPD (Random Amplification of Polymorphic DNA) (4) y el segundo espaciador intergénico ribosómico (ITS-2) (3), indican altos niveles de flujo génico entre las poblaciones domésticas, peridomésticas y silvestres del municipio de Boavita, Boyacá.

Además, el análisis del gen ITS-2 sugiere que las poblaciones colombianas pertenecen, junto con las de Panamá, a un mismo haplogrupo (denominado T. dimidiata capitata) (8,9). Este haplogrupo difiere significativamente con respecto a $T$. dimidiata de México y Centroamérica (Guatemala, Honduras, Nicaragua y Costa Rica) $(8,9)$. Estos trabajos demostraron que las cinco poblaciones continentales de Colombia presentan cinco haplotipos de ITS-2, con una gran diversidad haplotípica (Hd: 0,686 $\pm 0,065)$, pero con una muy baja diversidad de nucleótidos (п: 0,003 \pm 0,002) entre ellos (8). Tales valores indican que en Colombia el vector presenta una gran variabilidad genética, pero que el bajo nivel de diversidad molecular del ITS-2 limita la determinación de los posibles niveles de diferenciación genética de la población (8). Por tal razón, Bargues et al. (8) resaltan la necesidad de implementar un estudio de poblaciones con un mayor número de individuos de cada población, así como la aplicación de un nuevo marcador molecular con mayor nivel de variación, tal y como podrían ser los marcadores de tipo mitocondrial.

Teniendo en cuenta estos antecedentes, y dada la importancia epidemiológica de $T$. dimidiata en Colombia, consideramos que es necesario ampliar el conocimiento de la composición genética, el nivel de subdivisión y las dinámicas de las poblaciones, mediante la implementación de nuevos marcadores moleculares.

Uno de los marcadores moleculares mitocondriales que se ha destacado por su alto nivel polimórfico y que lo hace apropiado para los estudios de genética de poblaciones en insectos de importancia médica, es el gen de la subunidad 4 de la NADH 
deshidrogenasa (ND4) (10-12). Aunque existen más de 30 secuencias de ND4 depositadas en el GenBank, no existen estudios de poblaciones en triatominos con este marcador.

El presente trabajo pretendió determinar el nivel de variabilidad y diferenciación genética de tres poblaciones naturales de $T$. dimidiata de Colombia, mediante el análisis de un fragmento de 563 nucleótidos del gen mitocondrial ND4 en individuos capturados en palmas, rocas y áreas de influencia humana, como domicilios y peridomicilios.

\section{Materiales y métodos}

\section{Insectos}

Todos los individuos utilizados en este trabajo se identificaron morfológicamente como $T$. dimidiata (Latreille, 1811), según la clave taxonómica de referencia para triatominos (13). Se emplearon 40 individuos, 10 capturados en el departamento de La Guajira, 10 en el departamento de Santander y 20 en el departamento del Cesar. Los individuos silvestres de Santander fueron capturados en afloramientos de rocas, mientras los de La Guajira, en palmas $A$. butyracea. El número de ejemplares, su origen geográfico y el ecótopo de procedencia se muestran en el cuadro 1.

\section{Extracción de ADN, amplificación y secuenciamiento del ND4}

El ADN de cada insecto se extrajo de las patas, según Collins et al. (14). La reacción en cadena de la polimerasa (PCR) y el secuenciamiento del ND4 se realizaron según Lyman et al. (15), utilizando el iniciador ND4-F (5'TCAACATGAGCCCTTGGAAG-3') y ND4-R (5'TAATTCGTTGTCATGGTAATG-3'). Las secuencias de nucleótidos obtenidas se alinearon en ClustalX 1.81 (16), con un ajuste manual requerido usando el programa BioEdit, versión 7.0.9.0 (17). La primera posición del fragmento de 563pb del ND4 amplificado correspondió a la posición 8765 en el genoma mitocondrial de $T$. dimidiata (código de acceso, GenBank № AF301594) (18).

\section{Descripción y variabilidad del ND4}

Para cada población se estimó la composición nucleotídica promedio (porcentaje de adeninatimina), el número de sitios polimórficos, el número de sitios únicos (singleton sites), el número promedio de diferencias nucleotídicas y el número promedio de sustituciones de nucleótidos (transiciones y transversiones: ts/tv), utilizando los programas Mega 4.1 (19) y DnaSP 4.5 (20) (cuadro 1).
Se evaluaron dos estimadores de variabilidad genética: el índice de polimorfismo de nucleótidos $(\theta)$ y el índice de diversidad nucleotídica (п) (21), usando el programa DnaSP 4.5 (20). La asignación del número de haplotipos (h) y el cálculo de la diversidad haplotípica (Hd) (21), se llevaron a cabo usando los programas DAMBE 5.0.45 (22) y DnaSP 4.5 (20), respectivamente. La estimación de la distancia genética estándar ( $d$ ) (distancias genéticas dentro y entre poblaciones) se estimó mediante el modelo de Tamura-Nei (TrN) (23), usando el programa Mega 4.1 (19). Finalmente, para establecer conexiones en red (median joining networks) entre los haplotipos de las poblaciones, se usó el programa Network 4.5.1.0 (http://www. fluxus-engineering.com).

\section{Diferenciación de poblaciones}

El análisis jerárquico de varianza molecular (AMOVA) (24) se utilizó para probar la estructura de las poblaciones de $T$. dimidiata mediante el programa GenA1Ex 6.0 (25). El cálculo de la significancia $(5 \%)$ fue inferido a partir de las comparaciones por pares de los valores obtenidos de 1.000 permutaciones del estimador $\Phi_{\mathrm{ST}}$ (análogo del $\left.F_{S T}\right)$. Los valores del estimador $\Phi_{\text {ST }}$ de 0 a 0,05 indican poca diferenciación genética, de 0,05 a 0,15 una diferenciación moderada, de 0,15 a 0,25 una alta diferenciación, y los valores de más de 0,25 representan una diferenciación genética muy alta (26). El número promedio de haplotipos migrantes por generación $(\mathrm{Nm})$ entre las poblaciones se calculó utilizando el $\Phi_{\mathrm{ST}}$ como un análogo del $\mathrm{F}_{\mathrm{ST}}$ en la ecuación $N m=\left\{\left(1 / \Phi_{S T}\right)-1\right\} / 2$ (27) mediante el programa GenA1Ex 6.0 (25).

\section{Resultados}

Todos los insectos amplificaron una banda de $614 \mathrm{pb}$, correspondiente a 51 nucleótidos del gen ND4L y 563 del gen ND4. Las secuencias obtenidas del ND4 no mostraron inserciones, deleciones o codones de parada, que es lo esperado para genes codificantes mitocondriales. Todas las secuencias mostraron un alto porcentaje de adenina-timina, con variación entre 1,1\% y $1,5 \%$ en las poblaciones analizadas (cuadro 1).

El porcentaje de sitios polimórficos del ND4 fue de $15,27 \%$. Las secuencias de los individuos de Santander mostraron el mayor porcentaje de sitios polimórficos (S) (10,48\%), seguido de Cesar $(4,97 \%)$ y La Guajira $(0,53 \%)$ (cuadro 1 y figura 1). El número de mutaciones únicas (Su) y el promedio de cambios nucleotídicos (k) observado, 
fue comparativamente mayor en Santander, que en Cesar y La Guajira (cuadro 1 y figura 1).

Como era de esperarse en los organismos muy relacionados, el índice promedio de sustitución de nucleótidos para $T$. dimidiata reflejó una fuerte desviación hacia los cambios transicionales con respecto a los de transversión (ts/tv= 2,8) (cuadro 1). El $74 \%$ de los cambios de nucleótidos observados en la secuencia del gen ND4 analizada corresponden a transiciones.
Los valores estimados para el índice de polimorfismo $(\theta)$ y de diversidad nucleotídica (п) se muestran en el cuadro 1. Ambos índices mostraron ser comparativamente mayores en la población de Santander que en la de La Guajira y el Cesar, los cuales mostraron un nivel similar de polimorfismo entre sí, pero diferente diversidad en nucleótidos (cuadro 1).

Como era de esperarse para los marcadores mitocondriales con alto nivel de polimorfismo, se

Cuadro 1. Origen geográfico y estimadores de la variabilidad genética de un fragmento de gen mitocondrial ND4 en Triatoma dimidiata de Colombia.

\begin{tabular}{|c|c|c|c|c|c|c|c|c|c|c|c|c|c|}
\hline Departamento & $\begin{array}{l}\text { Municipio / } \\
\text { Localidad }\end{array}$ & $\begin{array}{l}\text { Latitud / } \\
\text { Longitud }\end{array}$ & Ecótopo & $n \% A T$ & s & Su & k & ts / tv & $\theta \pm$ de & $\pi \pm$ de & h & $\mathrm{Hd} \pm \mathrm{d}$ & \\
\hline La Guajira & $\begin{array}{l}\text { Dibulla/ } \\
\text { Gúmake }\end{array}$ & $\begin{array}{l}11^{\circ} 27^{\prime} 22^{\prime \prime} \mathrm{N} \\
-73^{\circ} 30^{\prime} 56^{\prime \prime} \mathrm{O}\end{array}$ & $\begin{array}{l}\text { Palma Attalea } \\
\text { butiracea }\end{array}$ & 10 & 75,2 & 3 & 1 & 1 & $1 / 0$ & $\begin{array}{l}0,019 \pm \\
0,001\end{array}$ & $\begin{array}{l}0,002 \pm \\
0,000\end{array}$ & 4 & $\begin{array}{l}0,644 \pm \\
0,152\end{array}$ \\
\hline Cesar & $\begin{array}{l}\text { Valledupar / } \\
\text { Seynimin }\end{array}$ & $\begin{array}{r}10^{\circ} 47^{\prime} 60^{\prime \prime} \mathrm{N} \\
-73^{\circ} 25^{\prime} 06^{\prime \prime} \mathrm{O}\end{array}$ & $\begin{array}{l}\text { Intradomicilio y } \\
\text { peridomicilio }\end{array}$ & 20 & 74,1 & 28 & 20 & 4 & $2 / 2$ & $\begin{array}{l}0,014 \pm \\
0,005\end{array}$ & $\begin{array}{l}0,006 \pm \\
0,002\end{array}$ & 8 & $\begin{array}{l}0,589 \pm \\
0,130\end{array}$ \\
\hline Santander & $\begin{array}{l}\text { Capitanejo/ } \\
\text { Chorreras }\end{array}$ & $\begin{array}{r}6^{\circ} 53^{\prime} 14^{\prime \prime} \mathrm{N} \\
-72^{\circ} 70^{\prime} 02^{\prime \prime} \mathrm{O}\end{array}$ & $\begin{array}{l}\text { Afloramientos } \\
\text { de rocas }\end{array}$ & 10 & 75,6 & 59 & 32 & 17 & $12 / 5$ & $\begin{array}{l}0,037 \pm \\
0,015\end{array}$ & $\begin{array}{l}0,031 \pm \\
0,007\end{array}$ & 10 & $\begin{array}{l}1,000 \pm \\
0,045\end{array}$ \\
\hline Total & & & & 40 & 74,8 & 86 & 31 & 19 & $14 / 5$ & $\begin{array}{l}0,036 \pm \\
0,011\end{array}$ & $\begin{array}{l}0,034 \pm \\
0,003\end{array}$ & 21 & $\begin{array}{l}0,863 \pm \\
0,048\end{array}$ \\
\hline
\end{tabular}

n: número de secuencias; \%AT: porcentaje de adenina-timina; S: número de sitios polimórficos; Su: número de sitios únicos (singleton sites); k: número promedio de diferencias nucleotídicas; ts/tv: número promedio de sustituciones nucleotídicas (transiciones y transversiones); $\theta$ : índice de polimorfismo nucleotídico; п: índice de diversidad nucleotídica; h: número de haplotipos; Hd: diversidad haplotípica; de: desviación estándar.

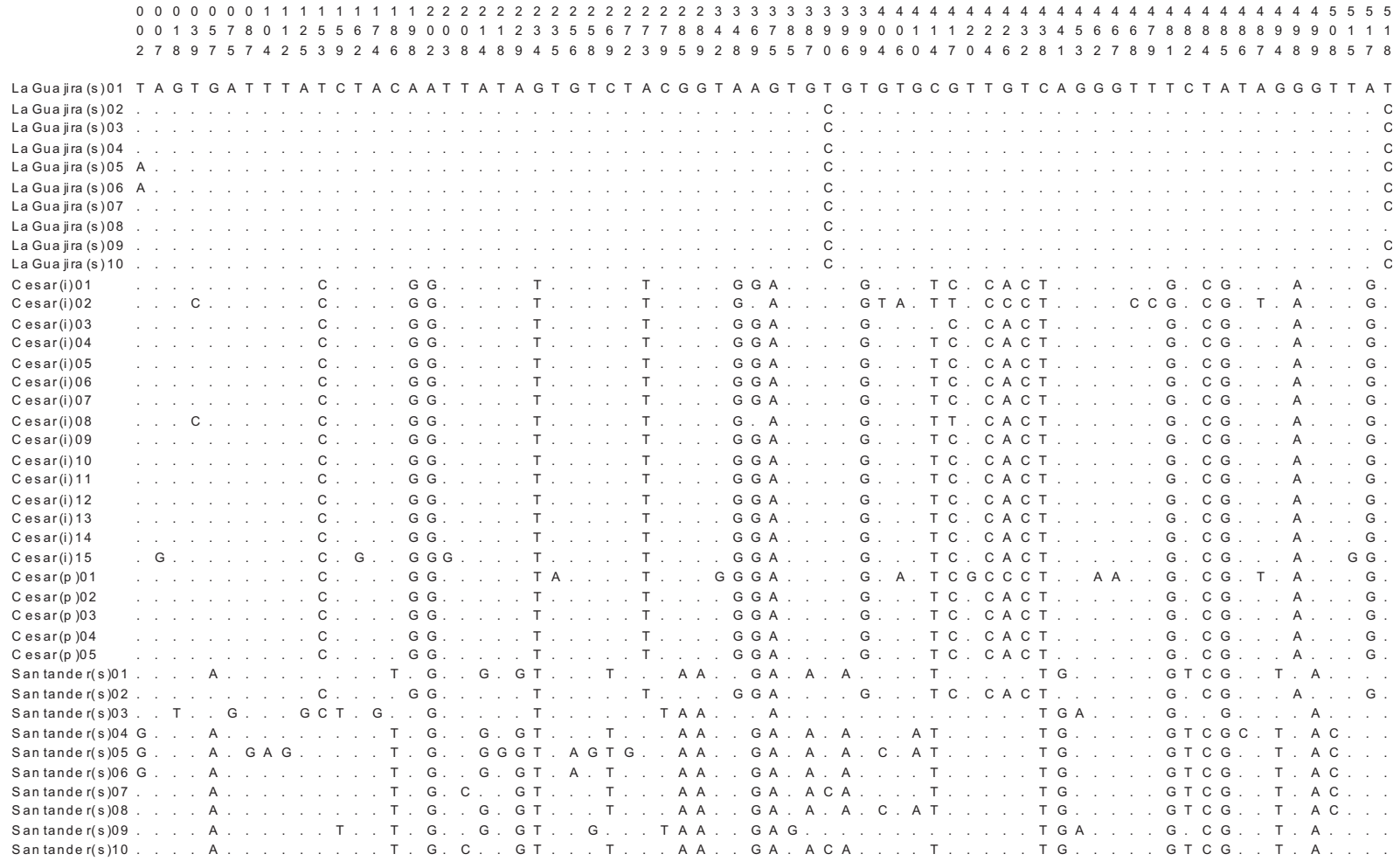

Figura 1. Alineamiento de 86 sitios polimórficos de un fragmento de 563 pb del gen ND4 en individuos de tres poblaciones colombianas de $T$. dimidiata. Los números sobre las secuencias indican las posiciones polimórficas entre las secuencias. Entre

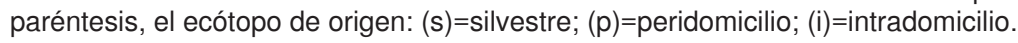


encontró un gran número de haplotipos (h) en $T$. dimidiata con una gran diversidad haplotípica (Hd) (cuadro 1). Todas las poblaciones mostraron haplotipos diferentes entre ellas, con excepción de un individuo de Santander que mostró un haplotipo similar al de algunos individuos de Cesar (véan se las secuencias de Santander 02 en la figura 1 y 2).

De forma similar a lo observado a nivel de nucleótidos, la diversidad de haplotipos de la población de Santander fue significativamente mayor que la del Cesar y La Guajira, los cuales mostraron niveles similares entre sí (cuadro 1).

La distancia genética $(\operatorname{TrN})$ promedio entre todos los individuos de $T$. dimidiata estudiados fue de $d=0,036$. La distancia estimada entre poblaciones sugiere una divergencia entre la población de Santander y la de La Guajira y el Cesar de 5,5\% y $5,4 \%$, respectivamente, y entre La Guajira y el Cesar, una divergencia del 4,5\%. La distancia estimada dentro de la población de Santander $(d=0,032)$ muestra un grado de divergencia, al menos, cinco veces mayor que la observada en Cesar $(d=0,006)$ y alrededor de 16 veces la de La Guajira $(d=0,002)$. La red de haplotipos muestra la agrupación de los individuos de las tres poblaciones que se corresponde con la distribución geográfica de las localidades de origen (figura 2). No se observó ninguna diferenciación entre los ecótopos de origen en la red haplotípica (figura. 2).

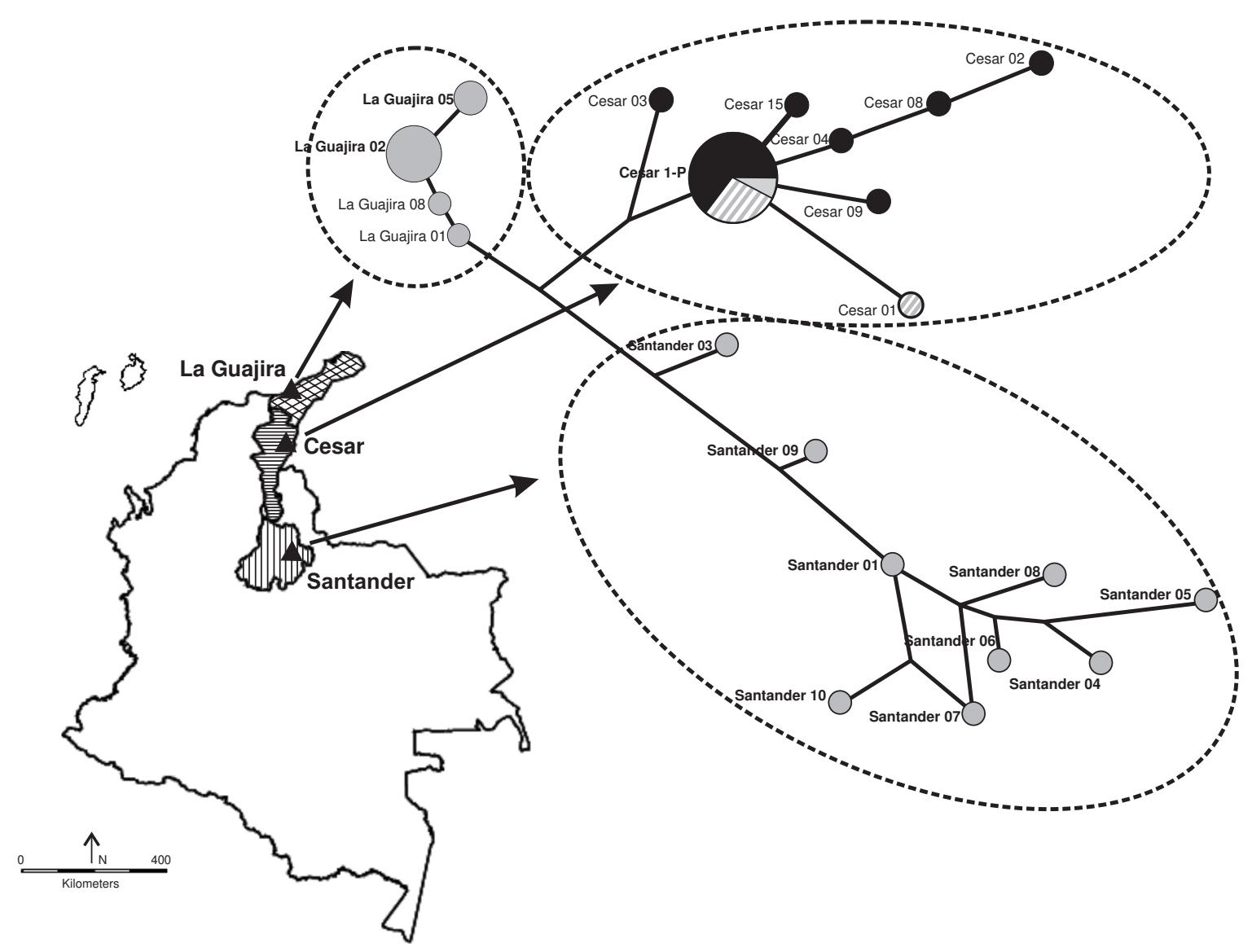

Diferenciación de las poblaciones de $T$. dimidiata en Colombia

Figura 2. Mapa de Colombia y análisis de conexiones haplotípicas (median-joining network) de tres poblaciones colombianas de $T$. dimidiata. El tamaño de los círculos representa la frecuencia haplotípica y los colores, el ecótopo de origen de los individuos. Gris: silvestre; franjas diagonales: peridomicilio y negro: intradomicilio. CES: Cesar; LAGUA: La Guajira y SANT: Santander.

CES1-P incluye las secuencias: Cesar(i)01, Cesar(i)05, Cesar(i)06, Cesar(i)07, Cesar(i)10, Cesar(i)11, Cesar(i)12, Cesar(i)13, Cesar(i)14, Cesar(p)02, Cesar(p)03, Cesar(p)04, Cesar(p)05, Santander(s)02; LAGUA02 incluye a La Guajira(s)03, La Guajira(s)04, La Guajira(s)07, La Guajira(s)09, La Guajira(s)10 y LAGUA05 incluye a La Guajira(s)06. Para mayor detalle acerca del origen y número de individuos analizados (cuadro 1). 
El AMOVA mostró que la variabilidad genética existente entre las tres poblaciones es, aproximadamente, tres veces mayor que la observada dentro de ellas (cuadro 2). El AMOVA permitió identificar una alta estructuración de haplotipos entre las poblaciones, con un bajo número de haplotipos migrantes por generación (cuadro 2). Igualmente, la comparación pareada de los valores del $\Phi_{S T}$ y la estimación del $\mathrm{Nm}$ entre las poblaciones, mostró en todos los casos un nivel de estructuración significativo y un restringido flujo de haplotipos migrantes, siendo particularmente mayor entre La Guajira y Cesar $\left(\Phi_{S T}=0,889\right.$; $N m=0,062)$, que entre La Guajira y Santander $\left(\Phi_{S T}=0,683 ; N m=0,232\right)$ y que entre Cesar y Santander $\left(\Phi_{S T}=0,696 ; N m=0,218\right)$.

\section{Discusión}

La variabilidad genética de $T$. dimidiata en Colombia se analizó inicialmente con ITS-2, incluyendo 30 individuos pertenecientes a poblaciones del centrooriente y del norte del país (8). Dicho trabajo mostró valores de diversidad de haplotipos ( $\mathrm{Hd}=0,686)$ y de nucleótidos $(\Pi=0,003)$ muy inferiores a los detectados por nosotros con ND4 ( $\mathrm{Hd}=0,863$; $\Pi=0,034)$.

Sin embargo, estos resultados eran de esperarse debido a que los genes mitocondriales poseen importantes diferencias con respecto a los nucleares, tales como tasas de mutación, herencia matrilineal y copias únicas, que pueden influir en la divergencia genética observada. Los valores de diversidad indican claramente que el gen mitocondrial ND4 es un marcador muy apropiado para el análisis de la genética de población de T. dimidiata en Colombia. Si tomamos en cuenta otros genes mitocondriales, la diversidad genética observada con el ND4 demostró ser similar a la reportada para el citocromo B en $T$. rubida $(\mathrm{Hd}=0,913 ; \Pi=0,033)$, especie filogenéticamente muy cercana a $T$. dimidiata y la cual presenta una compleja estructura de la metapoblación (28).

Cuadro 2. Resultado de la AMOVA para tres poblaciones colombianas de $T$. dimidiata basado en el análisis de un fragmento del gen mitocondrial ND4.

\begin{tabular}{lcccc}
\hline Fuente de variación & GL & $\begin{array}{c}\text { Porcentaje de } \\
\text { variación }\end{array}$ & 由ST & $\mathbf{N m}$ \\
\hline Entre poblaciones & 2 & 76 & $0,761^{\star * \star}$ & 0,157 \\
Dentro de poblaciones & 37 & 24 & & \\
Total & 39 & 100 & & \\
\hline${ }^{* *} \mathrm{p}<0,001$ & & & &
\end{tabular}

Por otra parte, y al igual que lo reportado para el ITS-2 (8), el análisis molecular de las secuencias de ND4 sugiere que tal variabilidad no es homogénea entre las poblaciones colombianas, ya que las del centro-oriente parecen presentar mayor diversidad génica que las de la región norte, lo cual permite, por tanto, determinar una clara estructuración de población.

Todos los índices de variabilidad genética estimados mostraron que la población de La Guajira posee un menor grado de ella que la de Cesar y la de Santander (cuadro 2). Esta diferencia genética dentro de la población podría relacionarse eventualmente con características ecológicas y geográficas particulares de cada región. El alto nivel de variabilidad mostrada en la población de Santander podría tener relación con la alta heterogeneidad ecológica observada en la región, en donde los individuos silvestres (que ocupan formaciones rocosas) y peridomésticos muestran una recurrente tendencia a la domiciliación $(3,4,6)$.

En el mismo sentido, el bajo grado de variabilidad de la población de La Guajira, podría deberse, quizá, al estricto hábitat arbóreo (palmas) que ocupa $T$. dimidiata en esta región de la vertiente norte de la Sierra Nevada de Santa Marta. Las diferencias genéticas observadas entre los individuos silvestres provenientes de afloramientos rocosos y los de palmas, sugiere que el origen ecológico de estos individuos podría jugar un papel preponderante en la subdivisión de poblaciones de $T$. dimidiata en Colombia.

Como se mencionó anteriormente, hasta el momento se han reseñado pocos casos de colonización de $T$. dimidiata en viviendas en la región norte de Colombia (5), pero éstos han sido transitorios (7). En este trabajo se incluyeron 15 individuos adultos recolectados en el intradomicilio y cinco en el peridomicilio de la localidad de Seynimin, ubicada en la vertiente sur-oriental de la Sierra Nevada de Santa Marta del departamento de Cesar. El análisis de estos ejemplares mostró una falta de diferenciación genética entre los individuos del intradomicilio y los del peridomicilio, lo que indicaría un proceso sostenible de dispersión entre ambos ecótopos.

Consideramos que es indispensable realizar más análisis sobre las tasas de migración entre las zonas y los ecótopos, para definir claramente la diferenciación genética y la capacidad de movilidad de $T$. dimidiata en Colombia. Igualmente, resaltamos 
la necesidad de realizar mayores acercamientos eco-epidemiológicos a $T$. dimidiata, a fin de diseñar estrategias apropiadas de intervención.

Otra característica importante de la población de Cesar es que, a pesar de ubicarse geográficamente más cerca a la localidad de Gúmake (Dibulla, La Guajira) que a la de Chorreras (Capitanejo, Santander), los niveles de diferenciación genética son suficientemente altos como para sugerir un origen de colonización distinto al de las poblaciones silvestres arbóreas de Gúmake.

En conclusión, los datos obtenidos en este trabajo describen, por primera vez, una subdivisión de poblaciones de $T$. dimidiata en Colombia, mediante la utilización del gen mitocondrial ND4. El entendimiento de la variabilidad biológica de este vector en el país es importante para poder proponer estrategias de control y vigilancia entomológica más focalizadas y efectivas. Por tal razón, se sugiere continuar con el análisis del gen mitocondrial ND4, incluyendo nuevas poblaciones, tanto en la región norte como la del centro-oriente de Colombia.

Las secuencias del ND4 usadas en este trabajo se encuentran disponibles en el GenBank con códigos de acceso GQ202151 - GQ202190.

\section{Conflicto de intereses}

Todos los autores de este trabajo declaramos que no existe conflicto de intereses.

\section{Financiación}

Este trabajo se realizó con el apoyo económico del CODI-Universidad de Antioquia (proyecto SIU- 21135), del Fondo para la Investigación y la Tecnología del Banco de la República (proyecto 2.233) y de Colciencias (proyectos 111545921460 y 3256-04-18067).

\section{Referencias}

1. Guhl F, Lazdins-Held J. Grupo de trabajo científico sobre la enfermedad de Chagas, Geneva: Special Programme for Research and Training in Tropical Diseases; 2007. p. 5-31.

2. Dorn P, Monroy C, Curtis A. Triatoma dimidiata (Latreille, 1811): A review of its diversity across its geographic range and the relationship among populations. Inf Gen Evol. 2007;7:343-52.

3. Bargues MD, GuhI F, Mas-Coma S. Genetic characterization of domestic, peridomestic and sylvatic Triatoma dimidiata populations from Colombia by ribosomal DNA ITS-2 sequence analyses. Acta Trop. 2002;83:149.

4. Ramírez C, Jaramillo C, Delgado P, Pinto N, Aguilera G, Guhl F. Genetic structure of sylvatic, peridomestic and domestic populations of Triatoma dimidiata (Hemiptera: Reduviidae) from an endemic zone of Boyacá, Colombia. Acta Trop. 2005;93:23-9.

5. Guhl F, Aguilera G, Pinto N, Vergara D. Actualización de la distribución geográfica y ecoepidemiología de la fauna de triatominos (Reduviidae: Triatominae) en Colombia. Biomédica. 2007;27(Suppl.1):143-62.

6. Angulo VM. Ensayo de estrategias de control y vigilancia de Triatoma dimidiata en Colombia. En: Guhl F, editor. Memorias del Primer Taller Internacional sobre Control de la Enfermedad de Chagas. Bogotá, D.C; Universidad de los Andes: 2005. p. 91-102.

7. Parra-Henao G, Angulo V, Jaramillo N, Restrepo M. Triatominos (Hemiptera: Reduviidae) de La Sierra Nevada de Santa Marta, Colombia. Aspectos epidemiológicos, entomológicos y de distribución. CES Med. 2009;23:17-26.

8. Bargues MD, Klisiowicz D, González-Candelas F, Ramsey JM, Monroy C, et al. Phylogeography and genetic variation of Triatoma dimidiata, the main Chagas disease vector in Central America, and its position within the genus Triatoma. PLoS Negl Trop Dis. 2008;2:e233. doi:10.1371/ journal.pntd.0000233.

9. Dorn PL, Calderón C, Melgar S, Moguel B, Solorzano E. Two distinct Triatoma dimidiata (Latreille, 1811) taxa are found in sympatry in Guatemala and Mexico. PLoS Negl Trop Dis. 2009;3:e393. doi:10.1371/journal.pntd.0000393.

10. Gorrochotegui-Escalante N, De Lourdes M, FernándezSalas I, Beaty B, Black W. Genetic isolation by distance among Aedes aegypti populations along the northeastern coast of Mexico. Am J Trop Med Hyg. 2000;62:200-9.

11. Uribe S, Lehmann T, Rowton E, Vélez I, Porter C. Speciation and population structure in the morphospecies Lutzomyia longipalpis (Lutz \& Neiva) as derived from the mitochondrial ND4 gene. Mol Phylogen Evol. 2001;18:8493.

12. Depaquit J, Lienard E, Verzeaux-Griffon A, Ferté $H$, Bounamous A, Gantier JC, et al. Molecular homogeneity in diverse geographical populations of Phlebotomus papatasi (Diptera: Psychodidae) inferred from ND4 mtDNA and ITS2 rDNA epidemiological consequences. Inf Gen Evol. 2008;8:159-70.

13. Lent $\mathbf{H}$, Wygodzinsky $\mathbf{P}$. Revision of the Triatominae (Hemiptera, Reduviidae) and their significance as vectors of Chagas disease. Bull Am Mus Nat His. 1979;163:123-520.

14. Collins FH, Méndez MA, Rasmussen MO, Mehaffey PC, Besansky NJ, Finnerty V. A ribosomal RNA gene probe differentiates member species of the Anopheles gambiae complex. Am J Trop Med Hyg. 1987;37:37-41.

15. Lyman D, Monteiro F, Escalante A, Cordon C, Wesson D, Dujardin J, Beard C. Mitochondrial DNA sequence variation among Triatominae vectors of Chagas disease. Am J Trop Med Hyg. 1999;60:377-86.

16. Thompson JD, Gibson TJ, Plewniak F, Jeanmougin F, Higgins DG. The Clustal $X$ windows interface: flexible strategies for multiple sequence alignment aided by quality analysis tools. Nucleic Acids Res. 1997;25:4876-82.

17. Hall T. BioEdit: a user-mendly biological sequences alignment editor and analysis program for Windows 95/98/ NT. Nucleic Acids Symp Ser. 1999;41:95-8. 
18. Dotson E, Beard C. Sequence and organization of the mitochondrial genome of the Chagas disease vector, Triatoma dimidiata. Insect Mol Biol. 2001;10:205-15.

19. Tamura K, Dudley J, Nei M, Kumar S. MEGA4: Molecular evolutionary genetics analysis (MEGA) software version 4.0. Mol Biol Evol. 2007;24:1596-9.

20. Rozas J, Sánchez-DelBarrio J, Messeguer X, Rozas R. DnaSP v 4.5, DNA polymorphism analyses by the coalescent and other methods. Bioinformatics. 2003;19:2496-7.

21. Nei M. Molecular evolutionary genetics. New York: Columbia University Press; 1987.

22. Xia X, Xie Z. DAMBE: Data analysis in molecular biology and evolution. J Hered. 2001;92:371-3.

23. Tamura K, Nei M. Estimation of the number of nucleotide substitutions in the control region of mitochondrial DNA in humans and chimpanzees. Mol Biol Evol. 1993;10:512-26.

24. Excoffier L, Smouse PE, Quattro JM. Analysis of molecular variance inferred from metric distances among
DNA haplotypes: application to human mitochondrial DNA restriction data. Genetics. 1992;131:479-91.

25. Peakall R, Smouse P. GENALEX 6: genetic analysis in Excel, population genetic software for teaching and research. Mol Ecol Notes. 2006;6:288-95.

26. Wright S. Evolution and the Genetics of Populations, vol. 4, Variability within and among natural populations. Chicago, IL: University of Chicago Press. 1978.

27. Frankham R, Briscoe D, Ballou J. Introduction to conservation genetics. New York: Cambridge University Press; 2002.

28. Pfeiler E, Bitler B, Ramsey J, Palacios-Cardiel C, Markow T. Genetic variation, population structure, and phylogenetic relationships of Triatoma rubida and $T$. recurva (Hemiptera: Reduviidae: Triatominae) from the Sonoran desert, insects vectors of the Chagas' Disease parasite Trypanosoma cruzi. Mol Phyl Evol. 2006;41:209-21. 